\title{
Robust Incremental SLAM under Constrained Optimization Formulation
}

\author{
Fang Bai, Teresa Vidal-Calleja, and Shoudong Huang
}

\begin{abstract}
In this paper, we propose a constrained optimization formulation of SLAM and a robust incremental SLAM framework. The new SLAM formulation is derived from the nonlinear least squares (NLS) formulation by mathematically formulating loop-closure cycles as constraints. Under the constrained SLAM formulation, we study the robustness of an incremental SLAM algorithm against local minima and outliers as a constraint/loopclosure cycle selection problem. We find a constraint metric that can predict the objective function growth after including the constraint. By the virtue of the constraint metric, we select constraints into the incremental SLAM according to a least objective function growth principle to increase robustness against local minima, and perform $\chi^{2}$ difference test on the constraint metric to increase robustness against outliers. Finally, using sequential quadratic programming (SQP) as the solver, an incremental SLAM algorithm (iSQP) is proposed. Experimental validations are provided to illustrate the accuracy of the constraint metric, and the robustness of the proposed incremental SLAM algorithm. Nonetheless, the proposed approach is currently confined to datasets with sparse loop-closures due to its computational cost.
\end{abstract}

Index Terms-SLAM, Localization, robust estimation, constrained optimization

\section{INTRODUCTION AND RELATED WORK}

$\mathbf{S}$ LAM as an optimization problem has been intensively studied during the past decade [1]. The current state-ofthe-art approach formulates SLAM as a NLS problem, which can be efficiently solved by local optimization techniques like Gradient Descent [2], Gauss-Newton [3][4] or its variations [5] by exploiting the matrix sparsity. The sparse NLS based solvers are rather efficient, however at the peril of occasionally converging to local minima (Fig. 1b). Another threat to SLAM comes from the incorrect loop-closures (outliers in black as shown in the second row of Fig. 11 typically produced by a front-end place recognition system. The problems of local minima and outliers are usually considered as two distinct problems, and a variety of remarkable literatures have been published to address either of the problems separately.

\section{A. Related Work}

A general strategy to tackle local minima is to use convex optimization techniques [6]. The techniques have been well

Manuscript received: September, 10, 2017; Revised December, 5, 2017; Accepted January, 3, 2018. This paper was recommended for publication by Editor Cyrill Stachniss upon evaluation of the Associate Editor and Reviewers' comments. The work is supported by University of Technology, Sydney and China Scholarship Council.

The authors are with the Centre for Autonomous Systems, University of Technology Sydney, Australia (e-mail: Fang.Bai@student.uts.edu.au; Teresa.VidalCalleja@uts.edu.au; Shoudong.Huang@uts.edu.au).

Digital Object Identifier (DOI): see top of this page.

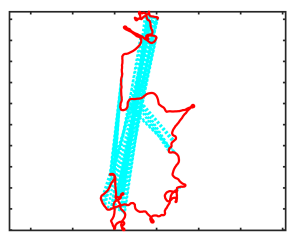

(a) Odometry.

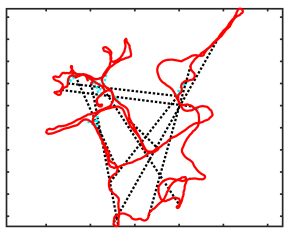

(d) iSQP (10 outliers).

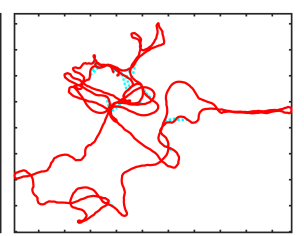

(b) Local minimum

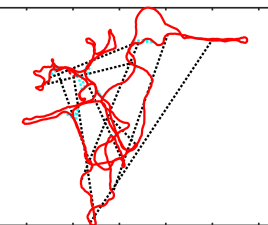

(e) DCS (10 outliers).

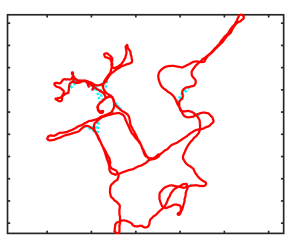

(c) Global minimum
Fig. 1. An example of how local minima and outliers jeopardize trajectory estimates on CSAIL_Pn dataset. The odometry is shown in (a) Initialized with the odometry, Gauss-Newton solver will converge to a local minimum as in (b) The global minimum in (c) can be obtained by using iSQP, or Mestimators like DCS or Cauchy (refined with Gauss-Newton). Nevertheless, in presence of only 10 outliers, DCS and Cauchy as shown in (e) and (f) cannot recover the correct trajectory given the poor odometry in (a) In this case, the proposed iSQP can recover the desired trajectory as shown in (d)

studied by the optimization community and some of them have already been employed in SLAM. The techniques can be used to obtain a good initial value for the optimizer that is sufficiently close to the global minimum of the original problem [7][8]. In some cases, like 2D SLAM, the convex relaxation can be very tight [9], thus the solution is accurate enough and can be used directly without any refinement. By replacing the angular distance of the rotation part in NLS with chordal distance, the objective function becomes quadratic. In this scenario, applying the Lagrangian dual theory, the global minimum can be verified or even computed from the dual problem if the duality gap is zero [10][11]. Recent progress [12][13] can efficiently provide certifiably global optimum using iterative Riemannian trust region methods. Another line of work stems from the idea that rather than solving the complicated full problem, it is better to divide it into several subproblems which are easier to solve and less likely to get trapped in local minima. Then the subproblems' solutions are combined together to obtain the solution for the original problem. The selection of measurements for the subproblems can be achieved spatially [14], temporally [4], or automatically controlled by a strategy, like weight functions in M-estimators [15][16].

To deal with outliers, despite the classical robust estimation theory from the optimization community [17], in SLAM, new insights and methods have been proposed over the past 
years. The first line of work introduces additional variables, either to the optimization problem [18] or to the probabilistic graphical models [19], to downweigh the influence of outliers. This line of work can be regarded as a variant/extension of M-estimators, as discussed in [19][20]. Since NLS SLAM formulation aims to minimize the residual error weighted by a covariance, the second line of work [21][20][22] uses the covariance explicitly to control the impact of outliers. In this sense, an outlier is regarded as the measurement extremely inconsistent with the noise model, thus the estimation algorithm adapts the covariance to make it fit the real measurement noise. The third line of work [23][24] exploits the probabilistic interpretation of the objective function, i.e., a $\chi^{2}$ distribution under Gaussian noise assumption. In these cases, by the idea that an outlier will lead to a larger $\chi^{2}$ error, the algorithm attempts to find a set of consistent measurements by performing $\chi^{2}$ tests with different strategies. The forth line of work formulates the outlier rejection problem as a measurement selection problem, then single cluster graph partitioning [25] or linear programming [26] is used to select a maximal set of consistent measurements. However, certain assumptions are made to formulate a tractable selection problem. Another line of work [27] generalizes multiple loop-closing hypotheses as a hyper-edge. Then a so-called "prefilter method" is used to reduce the hyper-graph to a standard pose-graph which will be further optimized with robust back-end techniques.

\section{B. Motivation and Contributions}

Can we formulate the problem of local minima and that of outliers as one problem, and solve both with a single method? It has been observed that M-estimators (which have been originally developed to tackle outliers) can be used to obtain a good initial value for optimizer in [15]. Further evidence is showed in [20][16] where dynamic covariance scaling (DCS) is proposed to deal with both local minima and outliers. In this paper, we propose a constrained optimization formulation for SLAM with a straightforward mathematical representation of loop-closure cycles as constraints. Then the problem of local minima and the problem of outliers are unified as a constraint/cycle selection problem.

To be specific, we formulate an incremental SLAM as a constraint/cycle selection problem. SLAM algorithms by incrementally feeding measurements into the solver have been shown to be robust against local minima [28][5]. Incremental approaches for outlier rejection have been proposed based on $\chi^{2}$ test [24]. In this paper, we firstly propose a constraint metric to predict the $\chi^{2}$ error change after including the constraint without actually solving the problem, then design an incremental SLAM framework based on least objective function growth principle and $\chi^{2}$ difference test.

The main contributions of our work are:

- A constrained pose SLAM formulation with mathematical representations of loop-closure cycles as constraints.

- A constraint metric that can predict the objective function change after including the constraint.

- An incremental SLAM algorithm robust against both local minima and outliers.

\section{Structure and Notations}

We will revisit the NLS SLAM formulation in Section II] and introduce the constrained SLAM formulation in Section III. The robustness of incremental SLAM as a constraint/cycle selection problem is discussed in Section IV. A SQP algorithm to solve the constrained SLAM formulation is given in Section $\mathrm{V}$. In Section VI, we present the constraint metric, an strategy to assess the constraint independence, and the incremental SQP SLAM. The experimental validation and discussions are provided in Section VII and VIII respectively. Section IX will conclude the paper.

Notations: In this paper, $\mathrm{SO}(3)$ denotes the specialorthogonal group which is a set of valid rotation matrices. The difference between two elements on $\mathrm{SO}(3)$ group is defined by the $\operatorname{logarithm}$ mapping $\log (\cdot)$. The homogeneous rigid transformation on robot poses can be described by the special Euclidean group (SE(3)) under group operation $\oplus$,

$$
\begin{gathered}
\oplus: \operatorname{SE}(3) \times \operatorname{SE}(3) \rightarrow \operatorname{SE}(3) \\
\left\{\mathbf{t}_{1}, \mathbf{R}_{1}\right\} \oplus\left\{\mathbf{t}_{2}, \mathbf{R}_{2}\right\}=\left\{\mathbf{t}_{1}+\mathbf{R}_{1} \mathbf{t}_{2}, \mathbf{R}_{1} \mathbf{R}_{2}\right\} .
\end{gathered}
$$

Let $\|\mathbf{e}\|_{\Sigma}^{2}=\mathbf{e}^{\top} \Sigma^{-1} \mathbf{e}$ be the squared Mahalanobis distance with covariance matrix $\Sigma$. Denote the rotation matrix sequential multiplication below as

$$
\prod_{i=m}^{n} \mathbf{R}_{i}=\mathbf{R}_{m} \cdot \mathbf{R}_{m+1} \cdots \mathbf{R}_{n} \quad\left(m, n \in \mathbb{Z}^{+}, m \leq n\right)
$$

where for the case $m>n, \prod_{i=m}^{n} \mathbf{R}_{i}=\mathbf{I}$.

\section{NLS Formulation OF POSE-GRAPH SLAM}

The structure of a pose-graph SLAM problem can be visualized as a directed graph $G(V, E)$ (Fig. 2a), where each robot pose corresponds to a node in the graph described by

$$
p_{i}=\left\{\boldsymbol{\Delta}_{i}, \mathbf{R}_{i}\right\} \in \mathbb{R}^{3} \times \operatorname{SO}(3), \quad i \in V .
$$

The theoretical transformation from $p_{i}$ to $p_{j}$ (i.e., the relative pose encoded to edge $(i, j) \in E)$ is given by $\varepsilon_{i, j}=\left\{\mathbf{t}_{j}^{i}, \mathbf{R}_{j}^{i}\right\}$, where $\mathbf{t}_{j}^{i}=\mathbf{R}_{i}^{\top}\left(\boldsymbol{\Delta}_{j}-\boldsymbol{\Delta}_{i}\right), \mathbf{R}_{j}^{i}=\mathbf{R}_{i}^{\top} \mathbf{R}_{j}$. Denote the noisy measurement of the relative pose by $\tilde{\varepsilon}_{i, j}=\left\{\tilde{\mathbf{t}}_{j}^{i}, \tilde{\mathbf{R}}_{j}^{i}\right\}$, then the measurement error can be defined as

$$
\mathbf{e}_{i, j}=\left[\begin{array}{c}
\mathbf{t}_{j}^{i}-\tilde{\mathbf{t}}_{j}^{i} \\
\log \left(\tilde{\mathbf{R}}_{j}^{i \top} \cdot \mathbf{R}_{j}^{i}\right)
\end{array}\right], \quad(i, j) \in E .
$$

The measurement noise is usually assumed to be zero-mean Gaussian, namely $\mathbf{e}_{i, j} \sim \mathscr{N}\left(\mathbf{0}, \Sigma_{i, j}\right)$. The NLS formulation of the pose-graph SLAM optimization problem is to find the best configurations of poses $p_{i}(i \in V)$ that minimize the sum of weighted measurement error squared

$$
\underset{p_{i}}{\arg \min } \sum_{i \in V}\left\|\mathbf{e}_{i, j}\right\|_{\Sigma_{i, j}}^{2}=\sum_{(i, j) \in E} \mathbf{e}_{i, j}^{\top} \cdot \Sigma_{i, j}^{-1} \cdot \mathbf{e}_{i, j} .
$$

Remark 1: We define the relative pose measurement error $\mathbf{e}_{i, j}$ using $\mathbb{R}^{3} \times \mathrm{SO}(3)$ instead of $\mathrm{SE}(3)$ directly such that the translation and rotation can be separated easily. This is useful to generalize the approach for point feature SLAM, although the math may not be as elegant as in SE(3).

Remark 2: In later discussion, we assume that there is a node index sequence such that all the noisy measurement $\tilde{\varepsilon}_{i, j}$ 


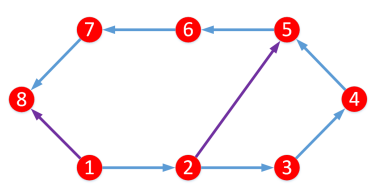

(a) A pose graph SLAM example with 8 poses, 7 odometry edges and 2 loop-closure edges

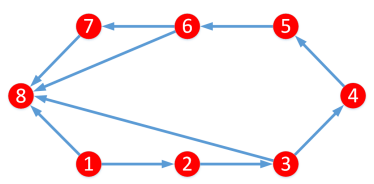

(c) Loop-closures to end-node 8 . $\left(\varepsilon_{2,5}\right.$ and $\left.\varepsilon_{1,8}\right)$.

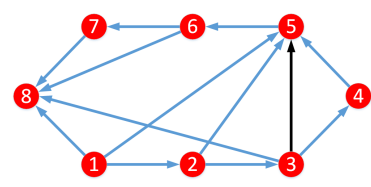

(b) The cycle $\varepsilon_{3,4}, \varepsilon_{4,5}, \varepsilon_{3,5}$ contains an outlier edge $\varepsilon_{3,5}$ which should not be included in the solver.

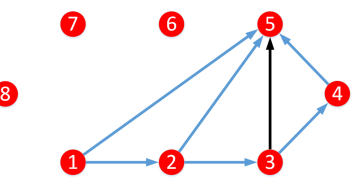

(d) Loop-closures to end-node 5.
Fig. 2. Diagram of loop closure cycles.

can be organized in the order $i<j$. If this does not hold, we invert the noisy measurement by

$$
\left\{\tilde{\mathbf{t}}_{j}^{i}, \tilde{\mathbf{R}}_{j}^{i}\right\}=\left\{\tilde{\mathbf{t}}_{i}^{j}, \tilde{\mathbf{R}}_{i}^{j}\right\}^{-1}=\left\{-\tilde{\mathbf{R}}_{i}^{j \top} \tilde{\mathbf{t}}_{i}^{j}, \tilde{\mathbf{R}}_{i}^{j \top}\right\} .
$$

While this inversion does not ensure exactly the same cost function as the original one, but in working precision the results are nearly the same according to our observations.

\section{CONSTRAined SLAM Formulation}

In SLAM, a complete loop-closure cycle includes the sequential transformations (odometry edges) and the loopclosure edge. For example in Fig. 2a odometry edges $\varepsilon_{2,3}$, $\varepsilon_{3,4}, \varepsilon_{4,5}$, and loop-closure edge $\varepsilon_{2,5}$ constitute a complete loop-closure cycle. In NLS formulation defined by (2), loopclosure cycles in the graph are not explicitly described.

\section{A. Mathematical Representation of A Loop-closure Cycle}

The sequential transformations of robot poses (i.e., edges in pose graph) can be explicitly described by a sequence of operations with the group operation $\oplus$ on $\operatorname{SE}(3)$.

In Fig. 2a, there are two loop-closure edges, $\varepsilon_{2,5}$ and $\varepsilon_{1,8}$. The complete loop-closure cycles corresponding to these two loop-closure edges can be described as below

$$
\begin{gathered}
\varepsilon_{2,3} \oplus \varepsilon_{3,4} \oplus \varepsilon_{4,5}=\varepsilon_{2,5} \\
\varepsilon_{1,2} \oplus \varepsilon_{2,3} \oplus \varepsilon_{3,4} \oplus \varepsilon_{4,5} \oplus \varepsilon_{5,6} \oplus \varepsilon_{6,7} \oplus \varepsilon_{7,8}=\varepsilon_{1,8} \\
\varepsilon_{1,2} \oplus \varepsilon_{2,5} \oplus \varepsilon_{5,6} \oplus \varepsilon_{6,7} \oplus \varepsilon_{7,8}=\varepsilon_{1,8} .
\end{gathered}
$$

Note loop-closure cycle (5) can be derived from loop-closure cycle (3) and (4), thus is redundant. In graph theory, a set of independent cycles that can be used to express all cycles in the graph are called "cycle basis" [29]. In Fig. 2a the cycle

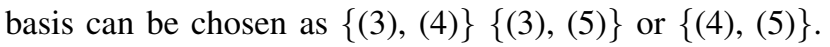

In general, we consider the case when: there are two edges (at least one loop-closure edge) to pose $p_{k}$ from pose $p_{i}$ and $p_{j}(j=i+m)$ when the robot travels from pose $p_{i}$ to $p_{j}$. The edges involved are $\varepsilon_{i, i+1}, \varepsilon_{i+1, i+2}, \cdots, \varepsilon_{i+m-1, i+m}$, and $\varepsilon_{i, k}$, $\varepsilon_{i+m, k}$. The loop-closure cycle is given in the form

$$
\varepsilon_{i, i+1} \oplus \varepsilon_{i+1, i+2} \oplus \cdots \oplus \varepsilon_{i+m-1, i+m} \oplus \varepsilon_{i+m, k}=\varepsilon_{i, k} .
$$

Further, with a slight abuse of notation, the information contained in 6 can be described as below two equations.

$$
\begin{array}{r}
{\left[\prod_{l=0}^{m-1} \mathbf{R}_{i+l+1}^{i+l}\right] \mathbf{t}_{k}^{i+m}+\sum_{t=0}^{m-1}\left[\prod_{l=0}^{t-1} \mathbf{R}_{i+l+1}^{i+l}\right] \mathbf{t}_{i+t+1}^{i+t}-\mathbf{t}_{k}^{i}=\mathbf{0}} \\
\log \left\{\left[\mathbf{R}_{k}^{i}\right]^{\top} \cdot\left[\prod_{l=0}^{m-1} \mathbf{R}_{i+l+1}^{i+l}\right] \mathbf{R}_{k}^{i+m}\right\}=\mathbf{0}
\end{array}
$$

The result can be easily obtained by expanding $\mathrm{SE}(3)$ group operation $\oplus$ and applying $\mathrm{SO}(3)$ logarithm mapping to the rotational part. We will refer to (7) and (8) as mathematical representations of loop-closure cycles in the following discussion.

Remark 3: The idea of considering a loop-closure as a cycle (i.e., the composition of relative poses) is not completely new. Olson [25] formulates two potential loop-closure edges along with the "Dijkstra link" as a loop to evaluate the pairwise consistency between the loop-closure edges. Dubbelman et al. [30] use relative transformations along a loop-closure cycle in their work "trajectory bending". Huang et al. [31] give a comprehensive discussion on the nonlinearity/convexity by using relative states in the formulation. Carlone et al. [32] exploit cycle basis to formulate the $2 \mathrm{D}$ orientation estimation problem as an integer optimization problem in terms of their regularizations.

\section{B. Constrained SLAM Formulation}

We propose a constrained SLAM formulation by taking all theoretical transformations $\varepsilon_{i, j},(i, j) \in E$ as variables to be estimated, and mathematical representations of loop-closure cycles (7) and (8) as equality constraints of the resulting optimization problem. From a graphical perspective, in graph $G$, we take all the edges as variables to be estimated, and constrain the problem with a cycle basis of $G$.

From (1) and (2), if we further define the weighted distance between the theoretical relative pose $\varepsilon_{i, j}$ and the noisy relative pose measurement $\tilde{\varepsilon}_{i, j}$ as

$$
\left.d\left(\tilde{\varepsilon}_{i, j}, \varepsilon_{i, j}\right)\right|_{\Sigma_{i, j}}=\mathbf{e}_{i, j}^{\top} \cdot \Sigma_{i, j}^{-1} \cdot \mathbf{e}_{i, j},
$$

then the objective function takes the form

$$
\left.d(\mathbf{x}, \eta)\right|_{\Sigma}=\left.\sum_{(i, j) \in E} d\left(\tilde{\varepsilon}_{i, j}, \varepsilon_{i, j}\right)\right|_{\Sigma_{i, j}}
$$

where $\mathbf{x}, \eta$, and $\Sigma$ collect all $\varepsilon_{i, j}, \tilde{\varepsilon}_{i, j}$, and $\Sigma_{i, j}$, respectively.

For convenience, we assign each loop-closure cycle an unique index, and use $\mathbf{C}_{i}(\mathbf{x})=\mathbf{0}$ to denote the constraint corresponding to the loop-closure cycle $i$. Compactly, the constrained SLAM formulation can be described as problem

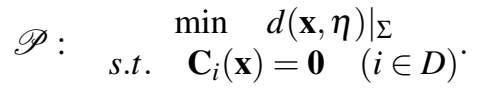

The indices of all the constraints are included in set $D$. Problem $\mathscr{P}$ can be solved by SQP (whose details will be provided in Section $\mathrm{V}$.

In short, the constraints are mathematical representations of loop-closure cycles, and the objective function can be regarded as a metric of the closeness between the estimated relative transformations and the actual noisy measurement data. In this 
sense, SLAM can be interpreted as finding the set of consistent transformations such that the constraints are satisfied, and meanwhile preserving the most information from the noisy measurement data.

Remark 4 (Observability): The NLS formulation (2) of SLAM is not observable, since the variables are absolute and the measurements are relative. As a result an anchor is required to solve the NLS formulation. However by using relative variables, the constrained SLAM formulation (9) is always observable thus the problem can be solved directly without introducing any anchor.

Remark 5 (Initial Value for Optimizer): The initial value for the iterative solver of the proposed constrained SLAM formulation (9) can be always chosen as the noisy measurement data $\eta$. Thus the initialization error is bounded by the measurement noise. This is different from the NLS formulation (2) where the initialization error is accumulated (e.g. through odometry or spanning tree).

Remark 6 (Relationship with NLS): In theory, the globally optimal solutions from both formulations are exactly the same. By applying a simple transformation it is possible to pass from one solution to the other and vice versa. For example, in the constrained formulation, after convergence where all constraints are satisfied, the absolute poses can be calculated by compounding the estimated state.

\section{The Case of Feature-based SLAM}

The feature based SLAM can be regarded as a special case of pose-graph SLAM, where some nodes as well as the edges to those nodes only contain the translational part. As a result, the constraints/loop-closure cycles in the feature based SLAM comprise only translational equations in the form of (7). This is the main reason why we keep translation and rotation separate. The feature based SLAM using constrained optimization has been studied by Bai et al. [33], but only the 2D outlier-free case is considered.

\section{RobUST InCREMENTAL SLAM AS A CONSTRAINT SELECTION PROBLEM}

In this section, we will discuss the robustness of an incremental SLAM algorithm towards local minima and outliers based on the constrained SLAM formulation.

\section{A. Incremental SLAM}

The key of an incremental SLAM algorithm is to design a sequence of subproblems $\mathscr{P}_{k}(k=0,1,2 \ldots n)$

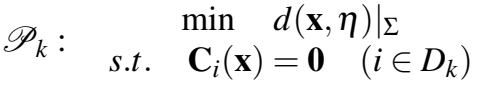

which only contains a portion of the constraints from the original problem $\mathscr{P}$. The sets of indices satisfy

$$
D_{0} \subset D_{1} \subset D_{2} \cdots D_{n-1} \subset D_{n} \subseteq D
$$

where $D_{0}=\emptyset$, indicating none of the constraints is included at subproblem $\mathscr{P}_{0}$.
When solving the subproblems sequentially with SQP,

$$
\mathbf{x}_{0} \underset{S Q P}{\stackrel{\mathscr{P}_{1}}{\longrightarrow}} \mathbf{x}_{1} \underset{S Q P}{\stackrel{\mathscr{P}_{2}}{\longrightarrow}} \mathbf{x}_{2} \cdots \mathbf{x}_{n-1} \underset{S Q P}{\stackrel{\mathscr{P}_{n}}{\longrightarrow}} \mathbf{x}_{n}
$$

we get a solution sequence $\mathbf{x}_{0}, \mathbf{x}_{1}, \mathbf{x}_{2} \ldots, \mathbf{x}_{n}$. If there are no outliers in the dataset, then $D_{n}=D$. If there are outliers in the dataset, then $D_{n}$ is designed to be the maximal constraint set which contains no outliers.

The most critical part of the incremental SLAM is how to design the constraint set $D_{k}$ for each subproblem $\mathscr{P}_{k}$, which can guarantee the global convergence between subproblems and ensure outlier free. In this sense, a robust incremental SLAM can be regarded as a constraint selection problem.

\section{B. Least Objective Function Growth (LOFG)}

To avoid local minima, the solution sequence $\left\{\mathbf{x}_{k}\right\}$ should be designed in a way that $\mathbf{x}_{k-1}$, i.e., the solution of subproblem $\mathscr{P}_{k-1}$ which also serves as the initial value of subproblem $\mathscr{P}_{k}$, lies within the basin of convergence of subproblem $\mathscr{P}_{k}$. To achieve this, one possible way is to ensure that $\mathbf{x}_{k-1}$ and $\mathbf{x}_{k}$ are as close as possible to each other as discussed by $\mathrm{Hu}$ et al. [15]. This heuristics can be described as a least solution change principle.

However, the solution change (the closeness of solutions) is not a well-defined concept since there are many potential criteria. In this paper, we use the objective function growth

$$
\Delta F_{k}^{k-1}=F_{k}-F_{k-1}=\left.d\left(\mathbf{x}_{k}, \eta\right)\right|_{\Sigma}-\left.d\left(\mathbf{x}_{k-1}, \eta\right)\right|_{\Sigma}
$$

between sequential subproblems to represent the solution change. As a result, the least solution change proposition becomes the least objective function growth (LOFG) principle.

\section{Constraint Metric}

The solution $\mathbf{x}_{k-1}$ is computed with the set of constraints in $D_{k-1}$, denoted by $\mathbf{x}_{k-1} \leftarrow\left\{\mathbf{C}_{i}\right\}_{i \in D_{k-1}}$. Incrementally, the solution $\mathbf{x}_{k}$ is computed from the set of constraints in $D_{k}=$ $D_{k-1} \cup\left\{i_{k}\right\}$, so $\mathbf{x}_{k}$ is dependent on $\mathbf{x}_{k-1}$ and $\mathbf{C}_{i_{k}}$, namely

$$
\mathbf{x}_{k} \leftarrow \mathbf{x}_{k-1} \cup \mathbf{C}_{i_{k}} \leftarrow\left\{\mathbf{C}_{i}\right\}_{i \in D_{k-1}} \cup \mathbf{C}_{i_{k}}
$$

The solution change from $\mathbf{x}_{k-1}$ to $\mathbf{x}_{k}$ is caused merely by the new added constraint $\mathbf{C}_{i_{k}}$. If the solution change is described by the objective function growth, is it possible to know the objective function growth $\Delta F_{k}^{k-1}$ prior to solving of subproblem $\mathscr{P}_{k}$ ?

Obviously, $\Delta F_{k}^{k-1}$ is related to the constraint $\mathbf{C}_{i_{k}}$ and the solution $\mathbf{x}_{k-1}$. Thus the problem becomes how to formulate a constraint metric, combined with solution $\mathbf{x}_{k-1}$, to predict the objective function growth $\Delta F_{k}^{k-1}$. Such a metric will be provided in Section VI-A

\section{D. $\chi^{2}$ Difference Test}

The validity of the objective function growth $\Delta F_{k}^{k-1}$ can be checked by $\chi^{2}$ difference test [34]. $\chi^{2}$ difference test is a variation of $\chi^{2}$ test employed on the difference of $\chi^{2}$ error and the change of the degree of freedom (DOF). In our case, after adding a constraint, the increase of $\chi^{2}$ error is $\Delta F_{k}^{k-1}$, 
meanwhile the DOF of constrained relative measurements rises from $n_{1}$ to $n_{2}$. Then the validity of $\Delta F_{k}^{k-1}$ can be examined by $\chi_{\alpha}^{2}\left(n_{2}-n_{1}\right)$ test, where $\alpha$ is the confidence probability which is usually set to 0.95 .

Different from existing methods in SLAM to deal with outliers [23][24], the change of $\chi^{2}$ error is predicted by a constraint metric, rather than solved from including the constraint. By the virtue of this, we perform $\chi^{2}$ difference test on the constraint metric. If the constraint metric passes the $\chi^{2}$ difference test, the loop-closure cycle implied by the constraint is considered to be outlier free; otherwise, containing outliers.

\section{E. Robustness}

The robustness of incremental SLAM against local minima and outliers stems from the fact that the constraint which satisfies the LOFG principle, in most of the cases, also passes the $\chi^{2}$ difference test. This is because a constraint satisfying LOFG principle is closer to an equality, thus the measurements constitute the loop-closure cycle are more consistent and unlikely to contain outliers. In other words, the LOFG principle and $\chi^{2}$ difference test, to some extent, correspond to how close the constraint added is to an equality, which motivates the constraint metric formulation in Section VI-A. Finally a robust incremental SLAM can be achieved by evaluating the constraint metric and select the constraint satisfying LOFG principle and $\chi^{2}$ difference test into the subproblems.

\section{IMPLEMENTATION OF SQP SOLVER}

In this section, we briefly introduce the implementation of SQP for constrained SLAM formulation.

\section{A. Sequential Quadratic Programming (SQP)}

SQP is realized by repeatedly performing linearization and solution update until the solution converges. For the constrained SLAM formulation in (9), the goal of linearization is to achieve a quadratic programming with a quadratic objective function and linear equality constraints

$$
\begin{array}{cl}
\min & \|\mathbf{H} \Psi-\eta\|_{\Sigma}^{2} \\
\text { s.t. } & \mathbf{A} \Psi=\mathbf{b}
\end{array}
$$

where the optimal solution $\Psi^{*}$ (and its covariance) can be computed by Lemma 1

Lemma 1: [35] The solution $\Psi^{*}$ to the quadratic programming $(12$ is

$$
\Psi^{*}=\gamma-\mathbf{Q A}^{\top}\left(\mathbf{A} \mathbf{Q} \mathbf{A}^{\top}\right)^{-1}[\mathbf{A} \gamma-\mathbf{b}]
$$

where $\mathbf{Q}=\mathbf{H}^{-1} \Sigma \mathbf{H}^{-\top}, \gamma=\mathbf{H}^{-1} \eta$. The uncertainty (covariance of Gaussian distribution) of $\Psi^{*}$ can be approximated by

$$
\operatorname{Cov}\left(\Psi^{*}\right)=\mathbf{Q}-\mathbf{Q} \mathbf{A}^{\top}\left(\mathbf{A} \mathbf{Q} \mathbf{A}^{\top}\right)^{-1} \mathbf{A} \mathbf{Q}
$$

Then the solution of the original problem (9) is updated by the quadratic programming solution $\Psi^{*}$ (which is usually an Euclidean increment) to obtain the new linearization point for the next iteration.

\section{B. Implementation on Manifold}

The key idea of optimization on $\mathbb{R}^{3} \times \mathrm{SO}(3)$ is to maintain a rotation matrix in the global scale which will be iteratively updated by Euclidean increments in the axis-angle space. The rotation matrix and axis-angle representation are related by exponential and logarithm mapping. The quadratic system (12) is attained by linearizing rotation matrices to the axis angles which then serve as the rotational part of the Euclidean increment. The details on how to compute the related Jacobian are provided in the supplementary material [36].

\section{ROBUST INCREMENTAL SQP SLAM}

In this section, we will formulate a constraint metric based on how close the constraint is to an equality. An approach to find independent constraints/loop-closure cycles (i.e., cycle basis in the graph) is also provided. Then we put things together and propose our robust incremental SQP SLAM.

\section{A. Constraint Metric}

From a probabilistic perspective, the closeness that a constraint is to an equality can be benchmarked by $f_{p d f}\left\{\mathbf{C}_{i}(\mathbf{x})=\right.$ $\mathbf{0}\}$, which is the probability density of $\mathbf{C}_{i}(\mathbf{x})$ at $\mathbf{0}$. However, it is difficult to obtain an exact analytical expression for the distribution of $\mathbf{C}_{i}(\mathbf{x})$, because of the nonlinearity introduced by rotation matrices. At $\mathbf{x}_{k-1}$, a viable approach is to find a Gaussian approximation for $\mathbf{C}_{i}(\mathbf{x})$ around $\mathbf{C}_{i}\left(\mathbf{x}_{k-1}\right)$.

At solution $\mathbf{x}_{k-1}$, constraint $\mathbf{C}_{i}(\mathbf{x})=\mathbf{0}$ can be linearized to $\overline{\mathbf{A}}_{i} \Psi=\overline{\mathbf{b}}_{i}$. Therefore the covariance can be approximated by $\overline{\mathbf{A}}_{i} \operatorname{Cov}\left(\Psi_{k-1}^{*}\right) \overline{\mathbf{A}}_{i}^{\top}$, where $\Psi_{k-1}^{*}$ is the Euclidean increment at subproblem $\mathscr{P}_{k-1}$. Hence, the Gaussian approximation for $\mathbf{C}_{i}(\mathbf{x})$ is given by

$$
\mathbf{C}_{i}(\mathbf{x}) \sim \mathscr{N}\left(\mathbf{C}_{i}\left(\mathbf{x}_{k-1}\right), \overline{\mathbf{A}}_{i} \mathbf{C o v}\left(\Psi_{k-1}^{*}\right) \overline{\mathbf{A}}_{i}^{\top}\right) .
$$

The probability density at $\mathbf{0}$ is

$$
\begin{aligned}
& f_{p d f}\left\{\mathbf{C}_{i}(\mathbf{x})=\mathbf{0}\right\} \\
& \propto \exp \left(-\mathbf{C}_{i}\left(\mathbf{x}_{k-1}\right)^{\top} \cdot\left[\overline{\mathbf{A}}_{i} \operatorname{Cov}\left(\Psi_{k-1}^{*}\right) \overline{\mathbf{A}}_{i}^{\top}\right]^{-1} \cdot \mathbf{C}_{i}\left(\mathbf{x}_{k-1}\right)\right) .
\end{aligned}
$$

During optimization, we use the negative logarithm of $f_{p d f}\left\{\mathbf{C}_{i}(\mathbf{x})=\mathbf{0}\right\}$, which is used as the constraint metric to predict the objective function growth $\Delta F_{k}^{k-1}$

$$
\Delta F_{k}^{k-1} \simeq \mathbf{C}_{i}\left(\mathbf{x}_{k-1}\right)^{\top} \cdot\left[\overline{\mathbf{A}}_{i} \operatorname{Cov}\left(\Psi_{k-1}^{*}\right) \overline{\mathbf{A}}_{i}^{\top}\right]^{-1} \cdot \mathbf{C}_{i}\left(\mathbf{x}_{k-1}\right) .
$$

Remark 7: The constraint metric (15) is a Mahalanobis distance incorporating the error and uncertainty accumulated in the loop-closure cycle, which is used in this paper to represent the impact of the loop-closure edge on the objective function. Similar ideas were used in EKF-based Mahalanobis gating to evaluate loop-closure hypotheses [37], where the motion uncertainty is accumulated in the robot pose.

\section{B. Independent Constraints/Loop-closure Cycles}

A typical method to decide the independence of cycles is to use Gaussian elimination, which has been used to find the minimum cycle basis of a graph [29]. The minimum cycle basis can ensure the sparsest system matrix, however is 


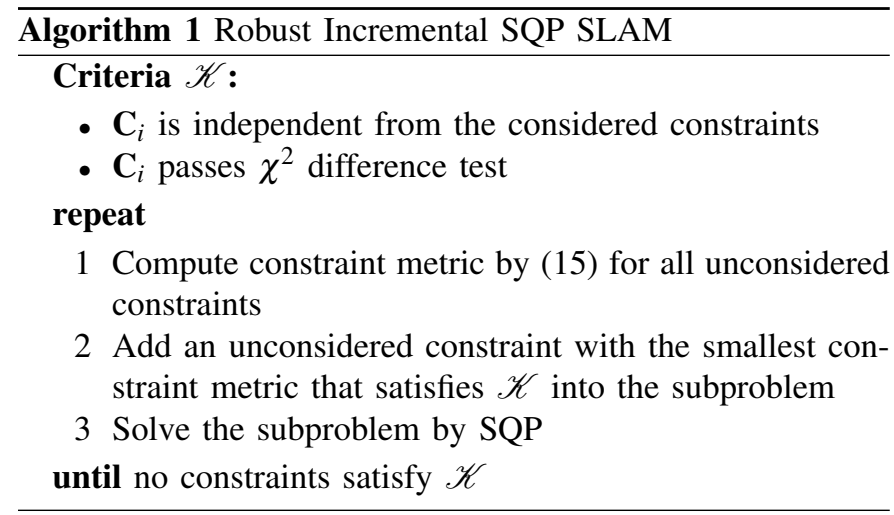

relatively expensive to compute. For simplicity, in this paper, we adopt a variation of the fundamental cycle basis [29], by assuming there exists an outlier free spanning tree, i.e., odometry, in presence of outliers (as shown in Fig. 2b].

Assume all the measurements are arranged from node $i$ to $j$, with $i<j$. In this case, the loop-closure cycles corresponding to different end-nodes are independent. For example, in Fig. $2 \mathrm{~b}$. there are two cluster of loop-closure cycles, with end-nodes 5 and 8 respectively. The loop-closure cycles corresponding to end-node 5 (shown in Fig. 2c) and that corresponding to endnode 8 (shown in Fig. 2d) are independent. For the cycles in each subgraph as in Fig. 2c and Fig. 2d, the independence of the cycles can be further assessed by Gaussian elimination or the methods described in the supplementary material [36].

\section{Robust Incremental SQP SLAM}

We propose a robust incremental SQP SLAM which summarizes the insights of the paper. The algorithm contains two components: constraint selection and SQP solver. For each subproblem, at a given solution, using constraint metric (15), the algorithm selects an independent constraint that can ensure LOFG, then followed by a $\chi^{2}$ difference test to identify whether the constraint is an inlier or not. After that, a SQP solver is used to solve the subproblem. The algorithm stops when all the independent constraints are considered (in the outlier free case), or all the unconsidered constraints fail $\chi^{2}$ difference test (in the case of presenting outliers).

The main procedure of the proposed robust incremental SQP SLAM is sketched in Algorithm 1 .

\section{RESULTS}

In this section, we will show the validity of the proposed framework, including the accuracy of the constraint metric and the robustness of iSQP.

\section{A. Evaluation Setup}

Since most mature techniques for outlier rejection are Mestimators or its variants [20][19], and it has been shown that M-estimators can work robustly against local minima as well, we compare with two of them in our experiments, dynamic covariance scaling (DCS) and Cauchy, which generalize the work in [18][20][16][15][19]. Both DCS and Cauchy are available in g2o implementation [3]. The tuning parameter for

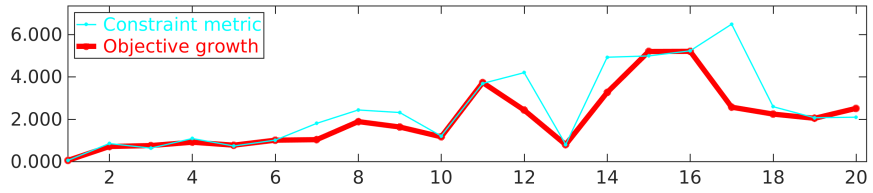

(a) MITb (Total 20 independent constraints).

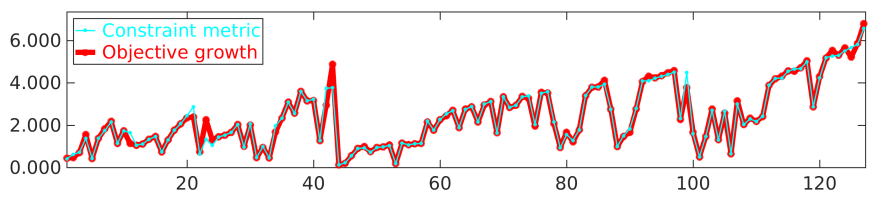

(b) CSAIL_Pn (Total 127 independent constraints).

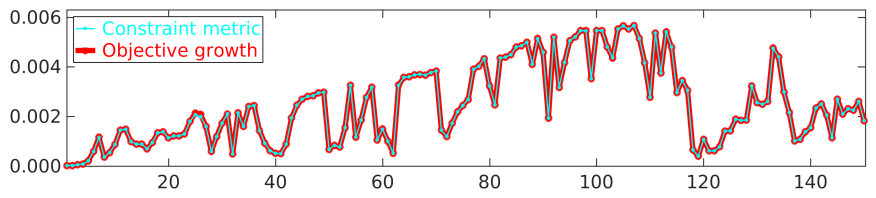

(c) Intel (Partial: first 150 independent constraints).

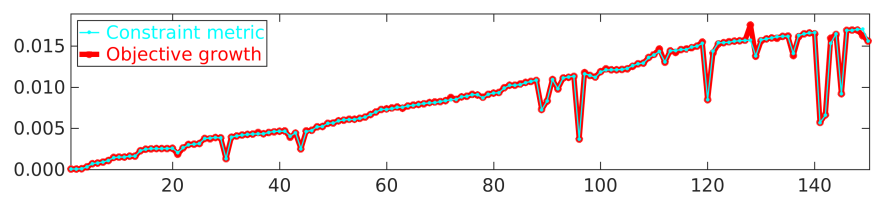

(d) Manhattan (Partial: first 150 independent constraints).

Fig. 3. The relationship between constraint metric and objective function growth. The root mean squared error from top to bottom are $0.2400,0.0168$, $7.2 \mathrm{e}-7$ and $1.5 \mathrm{e}-5$, respectively.

DCS can vary greatly depending on the dataset, so we perform an exhaustive search for the best tuning parameters within interval $[1,1000]$ for the datasets evaluated, which yields around 92 for MITb and 190 for CSAIL_Pn. For Cauchy, the tuning parameter is always set to 1 .

The outliers are generated by assigning the correct relative pose measurement computed from the global minimum estimate of two randomly selected poses, to two other poses. For the robustness measure, we use the absolute trajectory error (ATE), which is the mean of root squared position error of the estimated poses in presence of outliers with respect to that of optimal solution in the outlier free cases. The original datasets CSAIL_P, MITb, Intel, Manhattan used in the experiments can be found at [38].

\section{B. Accuracy of the Constraint Metric}

The constraint metric plays a key role in the proposed method. In this section, we show the accuracy of the metric (15) by solving 4 outlier free datasets with iSQP, and recording the constraint metric and corresponding objective function growth for each subproblem in Fig. 3 .

It can be seen from Fig. 3 that the proposed constraint metric (15) can generally follow the trend of the objective function growth, even for the noisy dataset MITb. The metric tends to be more accurate if the number of loop-closures in the dataset increases, for example CSAIL_Pn with 127 loopclosures. For less noisy and dense loop-closure datasets like Intel (with around 900 correct loop-closures) and Manhattan (with around 2000 correct loop-closures), the metric can nearly exactly predict the objective function growth. 


\section{Robustness of iSQP}

To show the robustness of iSQP against local minima and outliers, we firstly use a recreated dataset named CSAIL_Pn to show the point, then Monte Carlo simulation on MITb is used to test the potential of the algorithm.

1) CSAIL_Pn: CSAIL_Pn is a dataset recreated from CSAIL_P with increased noise, whose initial odometry is shown in Fig. 1a In this adverse case, Gauss-Newton will converge to a local minimum (Fig. 1b) while the global minimum can be attained by iSQP, DCS, or Cauchy (refined with Gauss-Newton) as shown in Fig. 1c In the presence of 10 outliers, both DCS (Fig. 1e) and Cauchy (Fig. 1f) would fail while iSQP is still robust (Fig. 1d).

2) Monte Carlo Simulation on MITb: We opt to use MITb for Monte Carlo simulation because: First, the constraint metric accuracy on MITb is worst among the 4 datasets in Section VII-B, which means it will be more challenging for iSQP. Second, the dataset is noisy, and 807 poses are sparsely connected by only 20 loop-closures. Therefore the problem is prone to converge to local minima and fail in presence of even 1 outlier. Third, the SQP solver is not very efficient, and the constraint metric is relatively expensive to compute. A Monte Carlo simulation on sparse loop-closures dataset will be faster, while still shows the point of our claim.

The initial odometry, local minimum and global minimum of the MITb dataset are plotted in the supplementary material [36]. In the outlier free case, a simple Gauss-Newton will converge to a local minimum, while the global minimum can be readily recovered by iSQP, or M-estimators refined with Gauss-Newton. Then we randomly add 1, 2, 5, 10, 15, 20 outliers to the dataset respectively, and run a 100 trial Monte Carlo simulation to test the robustness.

For comparison, we also use Cauchy+DCS combination (denoted by "Chy+DCS", with DCS tuning parameter 5) where Cauchy (denoted by "Chy") is used to bootstrap DCS. The ATE of different methods with respect to different number of outliers is depicted in Fig. 4. For DCS, Chy, and Chy+DCS, we define a trial to be a success if the ATE is less than 0.1. For iSQP, if all the outliers are identified and inliers are included, the solution is considered as a success. The averages of ATE and success rates (SR) for iSQP, DCS, Chy and Chy+DCS are summarized in Table I.

The result shows that: DCS can hardly converge for this noisy and sparsely connected dataset if initialized from odometry, while Cauchy can converge better but yield less accurate

TABLE I

PEFormance of ISQP, DCS AND CAUChy ON MITB Dataset

\begin{tabular}{cccccccc}
\hline \multicolumn{2}{l}{ Qty. of Outliers } & 1 & 2 & 5 & 10 & 15 & 20 \\
\hline \multirow{2}{*}{ iSQP } & ATE & 0.000 & 0.128 & 0.578 & 0.570 & 1.419 & 1.522 \\
& SR & $100 \%$ & $95 \%$ & $79 \%$ & $74 \%$ & $47 \%$ & $39 \%$ \\
\hline \multirow{2}{*}{ DCS } & ATE & 0.862 & 0.954 & 1.762 & 2.041 & 2.746 & 2.864 \\
& SR & $38 \%$ & $21 \%$ & $4 \%$ & $2 \%$ & $0 \%$ & $0 \%$ \\
\hline \multirow{2}{*}{ Chy } & ATE & 0.147 & 0.167 & 0.551 & 0.928 & 1.664 & 1.723 \\
& SR & $94 \%$ & $95 \%$ & $72 \%$ & $59 \%$ & $26 \%$ & $19 \%$ \\
\hline \multirow{2}{*}{ Chy- } & ATE & 0.153 & 0.182 & 0.509 & 0.821 & 1.429 & 1.464 \\
DCS & SR & $95 \%$ & $95 \%$ & $77 \%$ & $61 \%$ & $30 \%$ & $24 \%$ \\
\hline
\end{tabular}

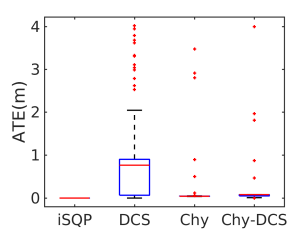

(a) 1 outlier.

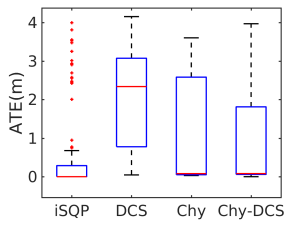

(d) 10 outliers.

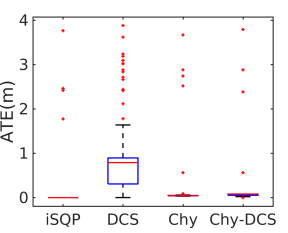

(b) 2 outliers.

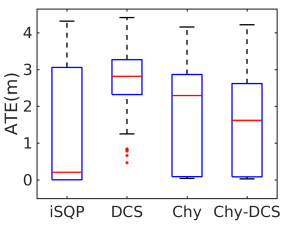

(e) 15 outliers

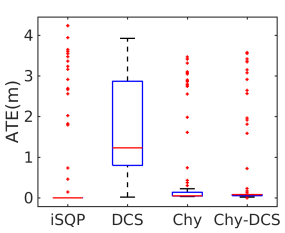

(c) 5 outliers.

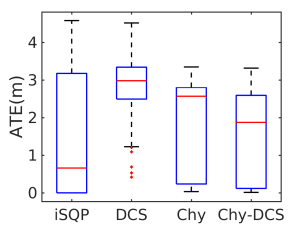

(f) 20 outliers.
Fig. 4. The ATE of iSQP, DCS, Cauchy and Cauchy+DCS combination in 100 run Monte Carlo simulation with respect to different number of outliers on the MITb dataset.

result. Cauchy+DCS combination outperforms both in terms of convergence and accuracy. However, iSQP can achieve comparable robustness, or slightly better in statistics.

\section{DISCUSSION}

M-estimators are famous for their simplicity and efficiency to deal with outliers. Nevertheless the outlier rejection is still embedded in the computation of the solution. An idea is to formulate the outlier rejection problem as a data selection problem [25][26] which is independent of the solver, thus the modularity of SLAM is enhanced. Motivated by previous work [15][25]|26]|27], we formulate a data selection problem to deal with both local minima and outliers. Even though LOFG principle is heuristic, the algorithm performs well in challenging sparsely connected pose-graph scenarios.

Recently, $\chi^{2}$ test is used as a benchmark to reject outliers in the graph SLAM formulation [23]|24]. However two problems remain. First, an overall $\chi^{2}$ test is not conspicuous, and the sensitivity decreases as the number of Gaussian measurements involved increases because high quality measurements can compensate poor quality measurements. The $\chi^{2}$ difference test is relatively more conspicuous compared with the $\chi^{2}$ test. Second, the existing methods need to solve the problem in order to obtain a $\chi^{2}$ error, which poses heavy burden to the solver and break the modularity of SLAM. By using a metric to predict $\chi^{2}$ error, the solver can maintain its efficiency and the modularity of SLAM is preserved.

The main issue of the proposed approach lies in its computational efficiency. For sparse dataset like MITb, it takes our Matlab implementation around $0.2 \mathrm{~s}$ for constraint selection and $2 \mathrm{~s}$ for subproblem resolution respectively on an Intel i5$5300 \mathrm{U} 2.3 \mathrm{GHz}$ quad-core CPU. However in adverse cases, the system matrix $\mathbf{A Q A}^{\top}$ in (13) (14) may lose sparsity, resulting in cubic (subject to matrix inversion) computational complexity. Another challenge for densely connected pose-graph datasets is that: if the noise is very large, a naive use of the metric (15) may not be robust enough. However, the method can be extended using joint validation, checking multiple cycles with respect to one single loop-closure edge. 


\section{CONCLUSION}

To summarize, we propose a constrained optimization formulation of pose-graph SLAM with mathematical representations of loop-closure cycles as constraints. We discuss the robustness of incremental SLAM as a constraint selection problem. We propose to use a constraint metric to predict the objective function growth, and select constraints according to the LOFG principle. An iSQP SLAM algorithm is proposed based on the SQP solver, and $\chi^{2}$ difference test. The results validate the accuracy of the constraint metric, and the comparable robustness of iSQP contrasted to DCS and Cauchy. The main issue of the proposed approach is its computational cost. In the future, we will work towards improvements in the efficiency of the solver, and more cost-effective constraint metrics.

\section{REFERENCES}

[1] C. Cadena, L. Carlone, H. Carrillo, Y. Latif, D. Scaramuzza, J. Neira I. Reid, and J. J. Leonard, 'Past, present, and future of simultaneous localization and mapping: Toward the robust-perception age" IEEE Transactions on Robotics, vol. 32, no. 6, pp. 1309-1332, 2016.

[2] E. Olson, J. Leonard, and S. Teller, 'Fast iterative alignment of pose graphs with poor initial estimates" in Robotics and Automation, 2006. ICRA 2006. Proceedings 2006 IEEE International Conference on. IEEE, 2006, pp. 2262-2269.

[3] R. Kümmerle, G. Grisetti, H. Strasdat, K. Konolige, and W. Burgard, "g2o: A general framework for graph optimization" in Robotics and Automation (ICRA), 2011 IEEE International Conference on. IEEE, 2011, pp. 3607-3613.

[4] M. Kaess, H. Johannsson, R. Roberts, V. Ila, J. J. Leonard, and F. Dellaert, "iSAM2: Incremental smoothing and mapping using the Bayes tree" The International Journal of Robotics Research, vol. 31, no. 2, pp. 216-235, 2012.

[5] D. M. Rosen, M. Kaess, and J. J. Leonard, "RISE: An incremental trustregion method for robust online sparse least-squares estimation " IEEE Transactions on Robotics, vol. 30, no. 5, pp. 1091-1108, 2014.

[6] S. Boyd and L. Vandenberghe, Convex optimization Cambridge university press, 2004.

[7] M. Liu, S. Huang, G. Dissanayake, and H. Wang, "A convex optimization based approach for pose SLAM problems" in Intelligent Robots and Systems (IROS), 2012 IEEE/RSJ International Conference on. IEEE, 2012, pp. 1898-1903.

[8] D. M. Rosen, C. DuHadway, and J. J. Leonard, 'A convex relaxation for approximate global optimization in simultaneous localization and mapping," in Robotics and Automation (ICRA), 2015 IEEE International Conference on. IEEE, 2015, pp. 5822-5829.

[9] L. Carlone, R. Aragues, J. A. Castellanos, and B. Bona, 'A fast and accurate approximation for planar pose graph optimization" The International Journal of Robotics Research, vol. 33, no. 7, pp. 965987, 2014

[10] L. Carlone, G. C. Calafiore, C. Tommolillo, and F. Dellaert, "Planar pose graph optimization: Duality, optimal solutions, and verification" IEEE Transactions on Robotics, vol. 32, no. 3, pp. 545-565, 2016.

[11] J. Briales and J. Gonzalez-Jimenez, "Fast global optimality verification in 3d slam," in Intelligent Robots and Systems (IROS), 2016 IEEE/RSJ International Conference on. IEEE, 2016, pp. 4630-4636.

[12] D. M. Rosen, L. Carlone, A. S. Bandeira, and J. J. Leonard, "A certifiably correct algorithm for synchronization over the special Euclidean group," in the Algorithmic Foundations of Robotics, The 12th International Workshop on, 2016.

[13] J. Briales and J. Gonzalez-Jimenez, "Cartan-sync: Fast and global se (d)synchronization," IEEE Robotics and Automation Letters, vol. 2, no. 4, pp. 2127-2134, 2017.

[14] G. Grisetti, R. Kümmerle, and K. Ni, 'Robust optimization of factor graphs by using condensed measurements" in Intelligent Robots and Systems (IROS), 2012 IEEE/RSJ International Conference on. IEEE, 2012, pp. 581-588.

[15] G. Hu, K. Khosoussi, and S. Huang, 'Towards a reliable SLAM back-end" in Intelligent Robots and Systems (IROS), 2013 IEEE/RSJ International Conference on. IEEE, 2013, pp. 37-43.
[16] P. Agarwal, G. Grisetti, G. D. Tipaldi, L. Spinello, W. Burgard, and C. Stachniss, 'Experimental analysis of dynamic covariance scaling for robust map optimization under bad initial estimates" in Robotics and Automation (ICRA), 2014 IEEE International Conference on. IEEE, 2014, pp. 3626-3631.

[17] P. J. Huber et al., "Robust estimation of a location parameter"' The Annals of Mathematical Statistics, vol. 35, no. 1, pp. 73-101, 1964.

[18] N. Sünderhauf and P. Protzel, "Towards a robust back-end for pose graph SLAM" in Robotics and Automation (ICRA), 2012 IEEE International Conference on. IEEE, 2012, pp. 1254-1261.

[19] G. H. Lee, F. Fraundorfer, and M. Pollefeys, 'Robust pose-graph loop-closures with expectation-maximization" in Intelligent Robots and Systems (IROS), 2013 IEEE/RSJ International Conference on. IEEE, 2013, pp. 556-563.

[20] P. Agarwal, G. D. Tipaldi, L. Spinello, C. Stachniss, and W. Burgard, "Robust map optimization using dynamic covariance scaling" in Robotics and Automation (ICRA), 2013 IEEE International Conference on. IEEE, 2013, pp. 62-69.

[21] E. Olson and P. Agarwal, 'Inference on networks of mixtures for robust robot mapping " The International Journal of Robotics Research, vol. 32, no. 7, pp. 826-840, 2013.

[22] M. C. Graham and J. P. How, 'Robust simultaneous localization and mapping via information matrix estimation" in Position, Location and Navigation Symposium-PLANS 2014, 2014 IEEE/ION. IEEE, 2014, pp. 937-944.

[23] Y. Latif, C. Cadena, and J. Neira, 'Robust loop closing over time for pose graph SLAM " The International Journal of Robotics Research, vol. 32, no. 14, pp. 1611-1626, 2013.

[24] M. C. Graham, J. P. How, and D. E. Gustafson, 'Robust incremental slam with consistency-checking." in Intelligent Robots and Systems (IROS), 2015 IEEE/RSJ International Conference on. IEEE, 2015, pp. 117-124.

[25] E. B. Olson, "Robust and efficient robotic mapping." Ph.D. dissertation, Massachusetts Institute of Technology, 2008.

[26] L. Carlone, A. Censi, and F. Dellaert, "Selecting good measurements via $\ell_{1}$ relaxation: A convex approach for robust estimation over graphs" in Intelligent Robots and Systems (IROS 2014), 2014 IEEE/RSJ International Conference on. IEEE, 2014, pp. 2667-2674.

[27] M. Pfingsthorn and A. Birk, "Generalized graph slam: Solving local and global ambiguities through multimodal and hyperedge constraints," The International Journal of Robotics Research, vol. 35, no. 6, pp. 601-630, 2016.

[28] M. Kaess, A. Ranganathan, and F. Dellaert, 'iSAM: Incremental smoothing and mapping" IEEE Transactions on Robotics, vol. 24, no. 6, pp. 1365-1378, 2008.

[29] T. Kavitha, C. Liebchen, K. Mehlhorn, D. Michail, R. Rizzi, T. Ueckerdt, and K. A. Zweig, "Cycle bases in graphs characterization, algorithms, complexity, and applications," Computer Science Review, vol. 3, no. 4, pp. 199-243, 2009.

[30] G. Dubbelman, I. Esteban, and K. Schutte, "Efficient trajectory bending with applications to loop closure," in Intelligent Robots and Systems (IROS), 2010 IEEE/RSJ International Conference on. IEEE, 2010, pp. 4836-4842

[31] S. Huang, Y. Lai, U. Frese, and G. Dissanayake, 'How far is SLAM from a linear least squares problem?' in Intelligent Robots and Systems (IROS), 2010 IEEE/RSJ International Conference on. IEEE, 2010, pp. 3011-3016

[32] L. Carlone and A. Censi, "From angular manifolds to the integer lattice: Guaranteed orientation estimation with application to pose graph optimization," IEEE Transactions on Robotics, vol. 30, no. 2, pp. 475492, 2014.

[33] F. Bai, S. Huang, T. Vidal-Calleja, and Q. Zhang, "Incremental SQP method for constrained optimization formulation in SLAM." in Control, Automation, Robotics and Vision (ICARCV), 2016 14th International Conference on. IEEE, 2016, pp. 1-6.

[34] K. Schermelleh-Engel, H. Moosbrugger, and H. Müller, "Evaluating the fit of structural equation models: Tests of significance and descriptive goodness-of-fit measures," Methods of psychological research online, vol. 8 , no. 2 , pp. $23-74,2003$

[35] J. Nocedal and S. J. Wright, Numerical optimization Springer, 2006.

[36] F. Bai, T. Vidal-Calleja, and S. Huang. Robust incremental SLAM under constrained optimization formulation. Supplementary material [Online]. Available: https://github.com/FangBaiUTS/ICRA2018

[37] I. J. Cox and S. L. Hingorani, "An efficient implementation of Reid's multiple hypothesis tracking algorithm and its evaluation for the purpose of visual tracking," IEEE Transactions on pattern analysis and machine intelligence, vol. 18, no. 2, pp. 138-150, 1996.

[38] [Online]. Available: www.lucacarlone.com/index.php/resources/datasets 